\title{
SCIENTIFIC REPORTS

\section{OPEN The possible role of Dickkopf-1, Golgi protein- 73 and Midkine as predictors of hepatocarcinogenesis: a review and an Egyptian study}

\author{
Abdel-Rahman N Zekri ${ }^{1 *}$, Mohamed EL Kassas ${ }^{2}$, El SayedTarekAbd El Salam ${ }^{3}$, \\ Reem Mostafa Hassan $\mathbb{D}^{4}$, Marwa Mohanad ${ }^{5}$, Reham Mohamed Gabr ${ }^{1}$, Mai M. Lotfy ${ }^{1}$, \\ Rania A. Talaat Abdel-zaher ${ }^{3}$, Abeer A. Bahnassy ${ }^{6}$ \& Ola S. Ahmed ${ }^{1}$
}

Hepatocellular carcinoma (HCC) is the second most common cause of cancer-related death worldwide. The use of alpha fetoprotein (AFP) alone was not an accurate biomarker for HCC despite its high specificity. Therefore, we assessed the possible role of serum biomarkers that have been mentioned briefly in previous studies on Egyptian patients ion top of HCV. However these studies included small number of patients and did not assess the different stages of hepatocarcinogenesis. In the current study we assessed 1) the expression levels of Golgi protein 37(GP73), Midkine (MDK) and Dickkopf1(DKK-1) proteins separately and in combination at different stages of hepatocarcinogenesis. GP73, MDK and DKK-1 proteins were assessed in 238 individuals divided into 4 groups (HCC, chronic HCV, and chronic HCV with cirrhosis and healthy subjects as a control) Serum levels of GP73, MDK, and DKK-1 were assessed in all subjects by ELISA. Serum levels of the studied markers were significantly higher in HCC compared to other groups ( $p<0.001)$. The ROC curve analysis for the studied markers showed 1$)$ 88.5\% sensitivity, $80.6 \%$ specificity, 69\% PPV, 93.5\% NPV and (AUC 0.91)for MDK; 2) 93.6\%, 86.9\%, 77.7\%, 96.5\% for DKK-1. 3) 91\%, 85\%, 74.7\%, 95\% (AUC 0.96) for GP73 and 4) 74.4\%, 84.4\%, 69.9\%, 87.1\% (AUC 0.81) for AFP. Serum levels of GP73, MDK, and DKK-1 are comparable to AFP as promising predictor biomarkers for HCC patients from Egypt. A two markers panel including Gp73 and DKK-1 showed the highest specificity and sensitivity among different markers combinations.

Hepatocellular carcinoma (HCC) is the second most common cause of cancer-related death worldwide and it was responsible for nearly 746,000 deaths in $2012^{1}$. Hepatitis C virus (HCV) infection has been considered the second most common risk factor of $\mathrm{HCC}^{2}$. Recent screening programs and the modern, highly sensitive imaging techniques used now such as ultrasound, CT and magnetic resonance imaging (MRI) have greatly improved early detection of HCC, which impacted positively on patients' outcome, mainly response to treatment and survival rates $^{3}$.

Serum biomarkers have also been used as a tool to screen and/or diagnose HCC at an early stage of the disease in several recent studies. Alpha-fetoprotein(AFP) is one of the thoroughly investigated biomarkers in different solid tumors, premalignant lesions or inflammatory conditions. It is a glycoprotein which is synthesized in large amounts by the yolk sac and liver during embryonic development. It disappears gradually after birth and is it only re-expressed in HCC. Therefore, it has been widely used clinically as a tumor marker for $\mathrm{HCC}^{4}$. The sensitivity and specificity for AFP varied widely in different studies at the early stages of hepatocarcinogenesis ${ }^{5}$. Therefore,

\footnotetext{
${ }^{1}$ Molecular Virology and Immunology Unit, Cancer Biology Department, National Cancer Institute, Cairo University, Cairo, Egypt. 'Endemic Medicine Department, Faculty of Medicine, Helwan University, Cairo, Egypt. ${ }^{3}$ Botany and Microbiology Department, Faculty of Science, Cairo University, Cairo, Egypt. ${ }^{4} \mathrm{Clinical}$ and Chemical Pathology Department, Faculty of Medicine, Cairo University, Cairo, Egypt. ${ }^{5}$ Biochemistry Department, College of Pharmaceutical Sciences and Drug Manufacturing, Misr University for Science and Technology, Giza, Egypt. ${ }^{6}$ Molecular pathology unit, pathology Department, National Cancer Institute, Cairo University, Cairo, Egypt. *email: ncizekri@yahoo.com
} 
identification of more reliable serum biomarkers; with high sensitivity and specificity; which complement AFP and improves clinical outcomes of those patients are highly required.

Recent studies have shown that other proteins could also be used as sensitive and specific markers for hepatitis as well as for HCC including the Golgi protein 73 (GP73); which is also named Golgi phosphoprotein 2 "GOLPH2, the Dickkopf-1(DKKpf-1 or DKK-1), and Midkine (MDK). These biomarkers have been used as potential, accurate, simple and non-invasive biomarkers for early detection of $\mathrm{HCC}^{6-8}$.

The GP73 is an occupant Golgi-specific membrane protein which is expressed by biliary epithelial cells in the normal liver. It increases in hepatitis, liver cirrhosis and maximally in HCC and it also accompanies the fibro-genesis stage (Maitra and Thuluvath, 2004) ${ }^{9}$. However, some studies demonstrated that GP73 serum level in HCC patients are markedly overlapped by the presence of cirrhosis, which affects its diagnostic accuracy ${ }^{10}$.

DKK-1 is a secretary protein with 266 -amino acid $(35 \mathrm{kDa})$ which is secreted directly into the blood stream. It is minimally expressed in the normal human adult tissues except the placenta and embryonic tissues. DDK1 increases in patients with various types of cancers including HCC, and therefore it could be used as a novel prognostic indicator in $\mathrm{HCV}$ - infected patients and it may also be used for early detection of $\mathrm{HCC}^{11}$.

Midkine (MDK) is a member of the heparin-restricting developmental factor family of proteins which was recognized as a cytokine in the HCV- associated HCC patients. It is expressed in several human malignancies including HCC among others. Some reports showed that the serum level of MDK is usually raised in most HCC cases, and that it may have an important role in AFP-negative patients as well as in early stage tumors ${ }^{12,13}$. Based on this data we sought to assess the possible diagnostic and prognostic roles of GP73, MDK, DKK proteins in the early detection of HCC in chronic HCV infected patients from Egypt.

\section{Methods}

Study design. This case control study included 238 participants who were admitted to the clinics of the National research center $(\mathrm{NRCH})$ and the National Cancer Institute (NCI), Cairo University during the period from November 2014 to October 2017.

The study protocol was approved by the institutional review board of the National Hepatology and Tropical Medicine Research Institute (NHTMRI) and the National Cancer Institute (NCI). The organization No. is IORG0003381 and the IRB NO. is IRB00004025). All steps of the research was performed in accordance with relevant guidelines (according to 2011 declaration of Helsinki). A written informed consent was obtained from each patient and control subject prior to enrollment in the study. AllHCC patients were diagnosed according to the American Association for the Study of Liver Diseases (AASLD) Practice Guidelines ${ }^{14}$ and staging was done based on Barcelona Clinic Liver Cancer (BCLC) staging system ${ }^{15}$.

Serum biomarkers in HCC patients were compared to chronic HCV patients either with or without liver cirrhosis as well as with apparently healthy donors as a control. All healthy individuals showed normal abdominal ultrasonography and no clinical or biochemical evidence of liver disease. HCV infection was confirmed in all cases by PCR using Artus HCV RT-PCR, QIAGEN PCR detection kit (Applied Bio systems 7500 fast system, ThermoFisher Scientific, USA) to detect HCV RNA expression levels. All patients were subjected to complete liver profile tests [including serum albumin, prothrombin time, bilirubin, and transaminases (ALT and AST)], complete blood picture (CBC), serum creatinine and Alfa fetoprotein using the automated biochemical Cobas 8000 modular analyzer c701/702, Roche, USA)]. Imaging studies included routine abdominal ultrasonography for in all subjects, and triphasic CT or dynamic MRI in patients with HCC. The exclusion criteria included patients with other liver diseases such as HBV co-infection and patients with a previous exposure to HCV antiviral therapy.

Sample preparation and measurement of serum biomarkers. Blood samples were collected from HCC patients at the time of diagnosis prior to surgery or any other treatment modalities. All serum samples were centrifuged, aliquoted and stored at $-80^{\circ} \mathrm{C}$ until being used for testing.

Assessment of the studied markers for all subjects was done using ELISA based kits (CLOUD CLONE CORP, Houstory, USA) according to manufacturers' instructions with optical density (O.D) measured at $450 \mathrm{~nm}$ wave length in a micro-plate reader (TECAN-Absorbance Reader INFNIT F50, Austria). The concentration of each protein was calculated according to manufacturers' instructions as follows;GP73 $(0.625-40 \mathrm{ng} / \mathrm{ml})$, MDK (15.625-1000 pg./ml), and DKK-1 (31.25-2000 pg/ml).

Statistical analysis. The SPSS, version 22.0 (IBM SPSS, Armonk, NY, USA) and graph-pad prism 7 were used for statistical analyses. The continuous variables were compared with one-way ANOVA with post-hoc Tukey test and Mann-Whitney U test was used to differentiate between the level of studied markers for each independent group. Chi-square was used for gender comparison among studied groups. The Receiver Operating Characteristic (ROC) curves were performed and Area under the curve (AUC) with 95\% CI was calculated to compare the diagnostic ability of each marker. Pearson's Chi-square correlation was used to assess the association between studied biomarkers and tumor size. Logistic regression model was used to assess the diagnostic performance of studied markers. All p-values are two-tailed and $\mathrm{P}<0.05$ was considered to indicate a statistically significant difference.

\section{Results}

Patient characteristics. A total of 238 participants were recruited in this study, including $78 \mathrm{HCV}$ - related HCC patients, 40 patients with HCV-related liver cirrhosis, and 40 chronic HCV patients without cirrhosis. Also included are 80 healthy individuals with no history of liver disease or alcohol consumption as a control group. The mean age for the studied participants was $48.5 \pm 14.7$ years. One hundred and fifty-fourpatients $(64.7 \%)$ were 


\begin{tabular}{|c|c|c|c|c|c|}
\hline & $\operatorname{HCC}(n=78)$ & Cirrhotic $(n=40)$ & $\begin{array}{l}\text { Non-cirrhotic } \\
(\mathrm{n}=40)\end{array}$ & $\begin{array}{l}\text { Control } \\
(\mathbf{n}=\mathbf{8 0})\end{array}$ & $\mathbf{P} *$ value \\
\hline \multicolumn{5}{|l|}{ Age } & \multirow{3}{*}{$<0.001$} \\
\hline mean $\pm S D$ & $58.27 \pm 9.7^{\mathrm{ab}}$ & $58.2 \pm 7.06^{\mathrm{ab}}$ & $50.7 \pm 10.25$ & $40.1 \pm 9.6$ & \\
\hline (range) & $(27-74)$ & $(40-69)$ & (29-69) & $(31-64)$ & \\
\hline \multicolumn{5}{|l|}{ Gender } & \multirow{3}{*}{$<0.001$} \\
\hline Male & $46(59)$ & $26(65)$ & $23(57.5)$ & $59(73.8)$ & \\
\hline Female & $32(41)$ & $14(35)$ & $17(42.5)$ & $21(26.2)$ & \\
\hline \multicolumn{5}{|l|}{ ALT } & \multirow{4}{*}{$<0.001$} \\
\hline Mean \pm SD & $68.36 \pm 34.1^{\mathrm{a}}$ & $80.37 \pm 57.8^{\mathrm{a}}$ & $42.9 \pm 20.5^{\mathrm{a}}$ & $22.9 \pm 6.0$ & \\
\hline (range) & $(12-140)$ & $(18-242)$ & $(14-330)$ & $(6-25)$ & \\
\hline Median & 50.5 & 50 & 42.5 & 12 & \\
\hline \multicolumn{5}{|l|}{ AST } & \multirow{4}{*}{$<0.001$} \\
\hline Mean \pm SD & $56.96 \pm 26.6^{\mathrm{ab}}$ & $66.5 \pm 46.9^{\mathrm{ab}}$ & $57.2 \pm 53.5^{\mathrm{a}}$ & $13 \pm 4.2$ & \\
\hline (range) & $(9-191)$ & $(19-335)$ & $(19-110)$ & $(12-40)$ & \\
\hline Median & 63.5 & 63.0 & 40.5 & 23.0 & \\
\hline \multicolumn{5}{|l|}{ Albumin } & \multirow{4}{*}{$<0.001$} \\
\hline Mean \pm SD & $3.4 \pm 0.53^{\mathrm{ab}}$ & $3.5 \pm 0.5^{\mathrm{ab}}$ & $4.0 \pm 0.68$ & $3.99 \pm 0.64$ & \\
\hline (range) & $(2.1-4.5)$ & $(2.8-4.7)$ & $(1.4-5.3)$ & $(1.2-4.9)$ & \\
\hline Median & 3.4 & 3.6 & 4.0 & 4.1 & \\
\hline \multicolumn{5}{|l|}{ AFP } & \multirow{4}{*}{$<0.001$} \\
\hline Mean \pm SD & $29.5 \pm 27.1^{1 \mathrm{abc}}$ & $13.6 \pm 11.99$ & $7.2 \pm 4.7$ & $5.6 \pm 1.9$ & \\
\hline (range) & $(1.4-100.0)$ & $(1.0-64.0)$ & $(10-20)$ & $(2.6-9.6)$ & \\
\hline Median & 18.9 & 10.0 & 6.0 & 5.5 & \\
\hline \multicolumn{5}{|l|}{$\mathrm{Hb}$} & \multirow{4}{*}{$<0.001$} \\
\hline Mean \pm SD & $12.34 \pm 1.6^{\mathrm{ab}}$ & $12.9 \pm 1.8^{\mathrm{a}}$ & $13.7 \pm 5.8$ & $14.2 \pm 1.3$ & \\
\hline (range) & $(9.1-15.5)$ & $(10.1-17.5)$ & $(11.4-16.4)$ & $(12.5-16.5)$ & \\
\hline Median & 12.6 & 13.0 & 13.6 & 13.9 & \\
\hline \multicolumn{5}{|l|}{ WBCs } & \multirow{4}{*}{$<0.001$} \\
\hline Mean \pm SD & $5.4 \pm 2.1^{\mathrm{a}}$ & $5.3 \pm 1.7^{\mathrm{a}}$ & $5.8 \pm 2.1^{\mathrm{a}}$ & $6.9 \pm 1.4$ & \\
\hline (range) & $(1.8-11.1)$ & $(2.6-9.3)$ & $(5.0-11.0)$ & $(4.2-10.0)$ & \\
\hline Median & 5.7 & 5.0 & 5.5 & 6.7 & \\
\hline \multicolumn{5}{|l|}{ Platelets } & \multirow{4}{*}{$<0.001$} \\
\hline Mean \pm SD & $127.4 \pm 62.8^{\mathrm{ab}}$ & $120.45 \pm 41.2^{\mathrm{ab}}$ & $216.3 \pm 55.5^{\mathrm{a}}$ & $312 \pm 85.1$ & \\
\hline (range) & $(3.8-308)$ & $(51.0-224.0)$ & $(88.0-356.0)$ & $(3.8-435.0)$ & \\
\hline Median & \begin{tabular}{|l|}
107.5 \\
\end{tabular} & 115.5 & 204.5 & 310.5 & \\
\hline
\end{tabular}

Table 1. The clinical feature of the studied groups. *ANOVA statistics, post-hoc tukey Gender wascompared using Chi-Square test. ${ }^{\mathrm{a}-\mathrm{c}}$ Groups bearing different initials are significantly different. ALT: Alanine aminotransferase; AST: Aspartate aminotransferase; AFP: $\alpha$ fetoprotein; Hb: hemoglobin; WBCs: White blood cells.

males and eighty-four (35.3\%) were females. The male to female ratio was (1.8: 1). Clinical features of the studied groups are shown in (Table 1).

The levels of serum biomarker in relation to tumor size. Serum levels of AFP, MDK, DKK-1, and GP73 were significantly higher in HCC compared to other studied groups (Table 2 and Fig. 1). However; there was no significant difference in serum concentration of the studied biomarkers among cirrhotic HCV patients, non-cirrhotic HCV patients and the healthy control group. Furthermore, the correlation between serum levels and tumor size in HCC patients was assessed as shown in Table 3 and Fig. 2. Serum levels of the four studied markers did not associate significantly with the size of the tumor.

Comparison between AUC, sensitivity, and specificity of the biomarkers for the diagnosis of HCC at optimal diagnostic cutoff values. The optimal diagnostic cut off values of AFP, MDK, DKK-1, and GP73 were determined using ROC curve analysis (Fig. 3). The cutoff value of AFP was $10.05 \mathrm{ng} / \mathrm{mL}$ with 0.81 AUC (95\% CI $0.74-0.88)$, 0.035SE, $74.4 \%$ sensitivity and $84.4 \%$ specificity. The optimal cut off for GK73 was $29.16 \mathrm{ng} / \mathrm{mL}$ with 0.956 (95\% CI $0.93-0.98)$ AUC, 0.014 SE, $91 \%$ sensitivity and $85 \%$ specificity $(\mathrm{P}<0.001)$. The optimal cut off for MDK, was $152.07 \mathrm{pg} / \mathrm{mL}$ with an AUC of 0.91 (95\% CI 0.88-0.95), SE of 0.019, a sensitivity of $88.5 \%$ and a specificity of $80.6 \%(\mathrm{P}<0.001)$. The cut off value of DKK1 was $344.8 \mathrm{pg} / \mathrm{mL}$ with an AUC of 0.956 (95\% CI $0.93-0.98)$, SE of 0.011 , a sensitivity of $93.6 \%$ and a specificity of $86.9 \%(\mathrm{P}<0.001)$. The predictive values, accuracy and likelihood ratios of all studied biomarkers for the diagnosis of HCC were calculated according to the 


\begin{tabular}{|c|c|c|c|c|c|c|c|}
\hline & Mean & Median & Range & IQR & 95\%CI & \multicolumn{2}{|l|}{ Subgroup P* value } \\
\hline \multicolumn{8}{|l|}{ AFP } \\
\hline $\mathrm{HCC}(\mathrm{n}=78)$ & $29.5 \pm 27.1$ & 18.9 & $1.4-100.0$ & 29.9 & $23.4-35.6$ & HCC vs C-HCV & $<0.001$ \\
\hline $\mathrm{C}-\mathrm{HCV}(\mathrm{n}=40)$ & $13.6 \pm 11.99$ & 10.0 & $1.0-64.0$ & 13.1 & $9.8-17.5$ & HCC vs NC-HCV & $<0.001$ \\
\hline NC-HCV $(n=40)$ & $7.2 \pm 4.7$ & 6.0 & $1.0-20.0$ & 7.0 & $5.7-8.7$ & HCC vs CON & $<0.001$ \\
\hline \multirow[t]{3}{*}{$\mathrm{CON}(\mathrm{n}=80)$} & $5.6 \pm 1.9$ & 5.5 & $2.6-9.6$ & 3.4 & $5.2-6$ & C-HCV vs NC-HCV & ns \\
\hline & & & & & & C-HCV vs CON & ns \\
\hline & & & & & & NC- $\mathrm{HCV}$ vs CON & ns \\
\hline \multicolumn{8}{|l|}{ GP73 } \\
\hline $\mathrm{HCC}(\mathrm{n}=78)$ & $105.97 \pm 112.1$ & 74.2 & $14.5-741.7$ & 70.9 & $80.7-131.2$ & HCC vs C-HCV & $<0.001$ \\
\hline $\mathrm{C}-\mathrm{HCV}(\mathrm{n}=40)$ & $14.66 \pm 7.45$ & 12.6 & $6.2-35.6$ & 6.4 & $12.2-17.0$ & HCC vs NC-HCV & $<0.001$ \\
\hline $\mathrm{NC}-\mathrm{HCV}(\mathrm{n}=40)$ & $18.9 \pm 9.6$ & 15.6 & $8.5-58.5$ & 10.8 & $15.8-22.0$ & HCC vs CON & $<0.001$ \\
\hline \multirow[t]{3}{*}{$\mathrm{CON}(\mathrm{n}=80)$} & $22.88 \pm 11.3$ & 21.7 & $5.53-77.7$ & 9.7 & $20.4-25.4$ & C-HCV vs NC-HCV & ns \\
\hline & & & & & & C-HCV vs CON & ns \\
\hline & & & & & & NC- $\mathrm{HCV}$ vs $\mathrm{CON}$ & ns \\
\hline \multicolumn{8}{|l|}{ MDK2 } \\
\hline $\mathrm{HCC}(\mathrm{n}=78)$ & $440.0 \pm 287.8$ & 382.4 & 103.77-1410.7 & 375.4 & 375.1-504.9 & HCC vs C-HCV & $<0.001$ \\
\hline $\mathrm{C}-\mathrm{HCV}(\mathrm{n}=40)$ & $168.72 \pm 167.7$ & 109.5 & $3.28-605.1$ & 155.7 & $115.1-222.4$ & HCC vs NC-HCV & $<0.001$ \\
\hline $\mathrm{NC}-\mathrm{HCV}(\mathrm{n}=40)$ & $59.9 \pm 89.5$ & 34.9 & $0.89-519.2$ & 65.1 & $31.3-88.6$ & HCC vs CON & $<0.001$ \\
\hline \multirow[t]{3}{*}{$\mathrm{CON}(\mathrm{n}=80)$} & $102.9 \pm 105.5$ & 76.8 & $0.1-517.1$ & 101.9 & $79.4-126.4$ & C-HCV vs NC-HCV & ns \\
\hline & & & & & & C-HCV vs CON & ns \\
\hline & & & & & & NC- $\mathrm{HCV}$ vs CON & ns \\
\hline \multicolumn{8}{|l|}{ DKK1 } \\
\hline $\operatorname{HCC}(\mathrm{n}=78)$ & $968.99 \pm 1240.6$ & 671.3 & $305.9-1044.29$ & 717.4 & \begin{tabular}{|l|}
$689.3-$ \\
1248.7
\end{tabular} & $\mathrm{HCC}$ vs $\mathrm{C}-\mathrm{HCV}$ & $<0.001$ \\
\hline $\mathrm{C}-\mathrm{HCV}(\mathrm{n}=40)$ & $272.8 \pm 153.2$ & 226.9 & $101.4-848.5$ & 129.6 & 223.9-321.9 & HCC vs NC-HCV & $<0.001$ \\
\hline $\mathrm{NC}-\mathrm{HCV}(\mathrm{n}=40)$ & $176.2 \pm 121.2$ & 153.7 & $20.2-803.8$ & 65.0 & $137.5-215.0$ & HCC vs Controls & $<0.001$ \\
\hline \multirow[t]{3}{*}{ Controls $(\mathrm{n}=80)$} & $247.3 \pm 128.5$ & 227.6 & $37.3-764.6$ & 172.1 & $218.7-275.9$ & C-HCV vs NC-HCV & ns \\
\hline & & & & & & C-HCV vs CON & ns \\
\hline & & & & & & NC- $\mathrm{HCV}$ vs CON & ns \\
\hline
\end{tabular}

Table 2. Serum biomarker levels in all studied groups. *Mann-Whitney U test. C-HCV: Cirrhotic hepatitis C, NC-HCV: non-cirrhotic hepatitis C., IQR: Interquartile range, CI: Confidence Interval, AFP: $\alpha$ fetoprotein, GP73: Golgi Protein 73, MDK: Midkine, DKKpf-1: Dickkopf-1 protein, ns: non-significant.

cut off values. The diagnostic accuracy of DKK1 (89.08\%) was the highest, followed by GP73 (87\%) then MDK (83.2\%). All three studied biomarkers had a diagnostic accuracy higher than AFP (81\%) (Figs. 3 and 4 \& Table 4).

The combination of studied biomarkers for the diagnosis of HCC. A binary logistic regression model was applied to assess the combinatorial ROC curves and to evaluate the diagnostic accuracy of the combinations of AFP, GP73, MDK and DKK3. The new variable predicted probability was created according to the equation obtained by binary logistic regression (HCC versus cirrhotic, non- cirrhotic and healthy controls. The model used in this study was as follows: for the combination of AFP and GP73, $\log [\mathrm{p} /(1-\mathrm{p})]=-6.79+(0.12 \times \mathrm{AFP})+$ $(0.125 \times \mathrm{GP73})$, for the combination of AFP and MDK, $\log [\mathrm{p} /(1-\mathrm{p})]=-3.61+(0.076 \times \mathrm{AFP})+(0.008 \times \mathrm{M}$ $\mathrm{DK})$, for the combination of AFP and DKK-1, $\log [\mathrm{p} /(1-\mathrm{p})]=-5.03+(0.066 \times \mathrm{AFP})+(0.008 \times \mathrm{DKK}-1)$, for the combination of MDK and GP73, $\log [\mathrm{p} /(1-\mathrm{p})]=-5.69+(0.103 \times \mathrm{GP73})+(0.005 \times \mathrm{MDK})$ for the combination of MDK and DKK- $1 \log [\mathrm{p} /(1-\mathrm{p})]=-4.88+(0.005 \times \mathrm{MDK})+(0.008 \times \mathrm{DKK}-1)$, for the combination of GP73 and DKK-1, $\log [\mathrm{p} /(1-\mathrm{p})]=-7.39+(0.099 \times \mathrm{GP73})+(0.007 \times \mathrm{DKK}-1)$, for the combination of AFP, GP73 and MDK, $\log [\mathrm{p} /(1-\mathrm{p})]=-7.21+(0.105 \times \mathrm{AFP})+(0.113 \times \mathrm{GP73})+(0.004 \times \mathrm{MDK})$, $)$, for the combination of AFP, MDK and DKK- $1, \log [\mathrm{p} /(1-\mathrm{p})]=-5.49+(0.065 \times \mathrm{AFP})+(0.005 \times \mathrm{MDK})+(0.007 \times \mathrm{DKK}-$ $1)$, for the combination of AFP, GP73 and DKK-1, $\log [\mathrm{p} /(1-\mathrm{p})]=-8.6+(0.097 \times \mathrm{AFP})+(0.106 \times \mathrm{GP} 73)+$ $(0.006 \times \mathrm{DKK}-1)$, for the combination of GP73, MDK and DKK-1, Log $[\mathrm{p} /(1-\mathrm{p})]=$ $-7.5+(0.095 \times \mathrm{GP73})+(0.002 \times \mathrm{MDK})+(0.007 \times \mathrm{DKK}-1)$ and for the combination of all four markers, Log $[\mathrm{p} /$ $(1-\mathrm{p})]=-8.62+(0.096 \times \mathrm{AFP})+(0.105 \times \mathrm{GP} 73)+(0.001 \times \mathrm{MDK})+(0.006 \times \mathrm{DKK}-1)$.

The new variable was used for ROC curve analysis in order to assess whether the combined use of AFP, GP73, MDK and DKK-1 was better than the use of any of these biomarkers alone. The AUC value for the combination of GP73 and DKK-1 was the highest $(0.987,95 \%$ CI $0.98-0.99)$ with $97.4 \%$ sensitivity and $93.1 \%$ specificity followed by AUC value for the combination of GP73 with AFP $(0.982,95 \%$ CI $0.97-0.99,96.2 \%$ sensitivity, $88.1 \%$ specificity) or $\operatorname{MDK}(0.975,95 \%$ CI $0.96-0.99,96.2 \%$ sensitivity, $87.2 \%$ specificity).

For triple markers combinations, the AUC was the largest for the combination of DKK-1 with GP73 and $\operatorname{AFP}(0.99,95 \%$ CI $0.99-1.0,98.7 \%$ sensitivity, $91.2 \%$ specificity) or with GP73 and MDK(0.99, 95\% CI 0.99-1.0, $98.7 \%$ sensitivity, $91.2 \%$ specificity). A similar result was shown for the combination of the four markers (AUC 0.99, 95\% CI 0.99-1.0, 98.7\% sensitivity, 91.2\% specificity) (Fig. 3, Table 4). These findings suggest that the three 

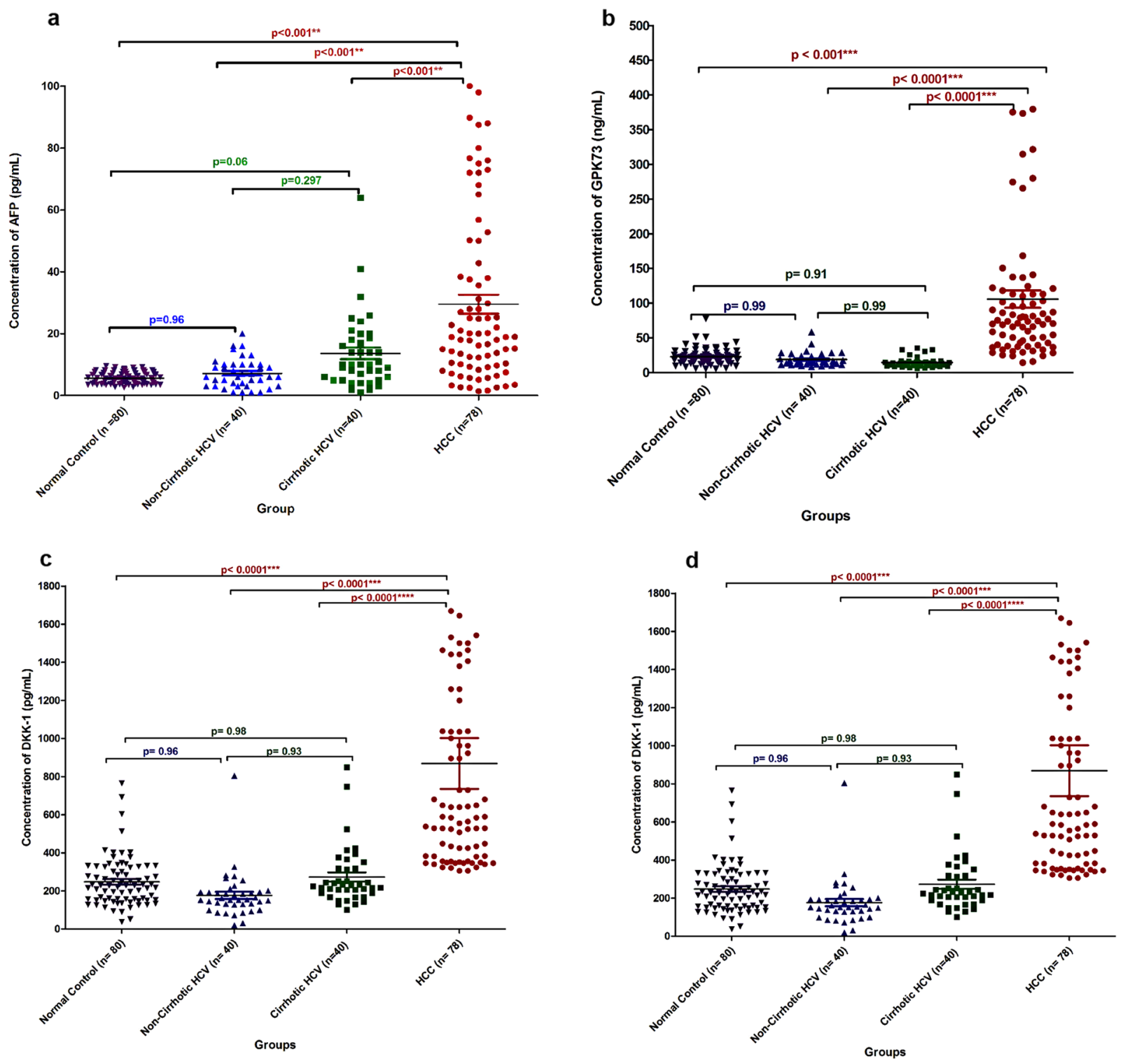

Figure 1. Scatter plots for (a) DDK-1, (b) GPK73, and (c) $M D K$ Levels are presented as $\mathrm{ng} / \mathrm{ml}$ in hepatocellular carcinoma, cirrhotic, chronic hepatitis and healthy controls.

markers panel (GP73, MDK and DKK-1) with or without AFP could improve the diagnostic efficacy for discriminating HCC patients from cirrhotic and non-cirrhotic hepatitis $\mathrm{C}$ virus carriers.

Correlation of studied markers in all studied groups and in HCC. In all studied groups, there was strong positive correlation between MDK and GP73 $(\mathrm{r}=0.612 ; \mathrm{p} .<0.001)$. In addition, DKK-1 was positively correlated to GP73 $(r=0.282, \mathrm{p}<0.001)$ and MDK $(\mathrm{r}=0.547, \mathrm{p}<0.001)$. In HCC group, serum MDK levels were significantly correlated to GP73 $(\mathrm{r}=0.441, \mathrm{p}<0.001)$ and to DKK-1 $(\mathrm{r}=0.482, \mathrm{p}<0.001)$. However, no significant correlation was detected between GP73 and DKK1 $(r=0.066, p=0.565)$ (Fig. 5 \& Table 5).

In the HCC group, there was a moderately positive correlation between MDK\& DDK-1 $(\mathrm{r}=0.0441)$ as well as between MDK and GP73( $\mathrm{r}=0.482 ; \mathrm{p}<0.001)$. On the other hand, either a weakly positive correlation or no correlation was detected between MDK, and GP73 $[\mathrm{r}=0.066 ; \mathrm{p}=0.565$; rho value $<0.2$; (Supplement-1) No significant correlation was found between GP73\& DKK-1 $(\mathrm{r}=0.066 ; \mathrm{p}=0.565)$.

On the other hand, our data did not showed any significant correlation between tumor size and all tested markers contrasts with some previous data in literature which showed that DKK-1 protein level correlates significantly with tumor size especially in HCV and/or HBV infected(Supplement-2).

\section{Discussion}

In the last few years many proteins have been addressed as potential biomarkers for diagnosing HCC that replaced AFP in order to increase its diagnostic accuracy, either alone or combined with it. Some of these markers are increasingly used in the routine workup of the patients such as Lectin-bound alpha-fetoprotein (AFP-L3) and 


\begin{tabular}{|c|c|c|c|c|c|}
\hline \multirow{2}{*}{\begin{tabular}{|l} 
Marker \\
\end{tabular}} & \multicolumn{4}{|c|}{ Tumor size $(\mathrm{cm})$} & \multirow{2}{*}{\begin{tabular}{|l|} 
P*value \\
0.21 \\
\end{tabular}} \\
\hline & & $<2(\mathrm{n}=25)$ & $2-3(n=35)$ & $>3(\mathrm{n}=16)$ & \\
\hline \multirow{4}{*}{ AFP } & Mean \pm SD & $36.1 \pm 31.4$ & $23.7 \pm 23.1$ & $30.0 \pm 26.1$ & \\
\hline & Median & 18.9 & 16.6 & 25.2 & \\
\hline & Range & $\mid 1.4-89.8$ & $2.5-98.0$ & $3.0-100.0$ & \\
\hline & $95 \% \mathrm{CI}$ & $23.2-49.1$ & \begin{tabular}{|l|}
$15.8-31.7$ \\
\end{tabular} & $16.1-43.9$ & \\
\hline \multirow{4}{*}{ GP73 } & Mean \pm SD & $105.7 \pm 90.8$ & $119.9 \pm 142.3$ & $80.9 \pm 63.7$ & 0.53 \\
\hline & Median & 86.4 & 75.5 & 61.7 & \\
\hline & Range & $28.5-379.9$ & $14.5-741.7$ & $16.1-265.8$ & \\
\hline & $95 \% \mathrm{CI}$ & $68.1-143.1$ & $71.0-168.8$ & 47.0-114.9 & \\
\hline \multirow{4}{*}{ MDK } & Mean \pm SD & $386.9 \pm 272.6$ & $466.9 \pm 288.1$ & $474.0 \pm 331.3$ & 0.52 \\
\hline & Median & 314.3 & 426.2 & 409.6 & \\
\hline & Range & $105.8-1102.1$ & $103.8-1410.7$ & $106.2-1371.8$ & \\
\hline & $95 \% \mathrm{CI}$ & \begin{tabular}{|l|}
$274.4-499.4$ \\
\end{tabular} & \begin{tabular}{|l|}
$367.9-565.9$ \\
\end{tabular} & $297.5-650.5$ & \\
\hline \multirow{4}{*}{ DKK-1 } & Mean \pm SD & $761 \pm 383$ & $1187.1 \pm 1789.9$ & $875.4 \pm 427.2$ & 0.41 \\
\hline & Median & 729.2 & 680.5 & 762.2 & \\
\hline & Range & $317.8-1541.5$ & $305.9-110442.1$ & $346.4-1658.1$ & \\
\hline & 95\% CI & 602.7-919.4 & 572.4-1802.0 & $647.7-1103.0$ & \\
\hline
\end{tabular}

Table 3. Correlation between serum levels of MDK, DKKpf-1, Gp73 and tumor sizes in patients with AFP inHCC infection. *ANOVA for association of serum markers levels with tumor size. AFP: $\alpha$ fetoprotein, GP73: Golgi Protein 73, MDK: Midkine, DKKpf-1: Dickkopf-1 protein.

a

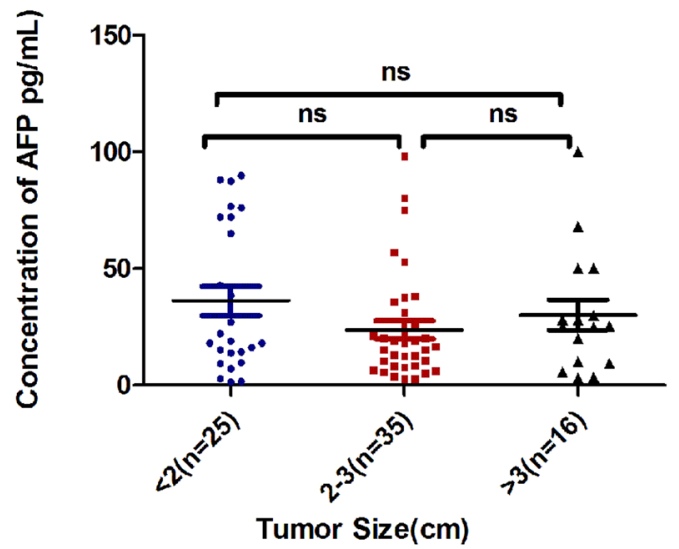

C

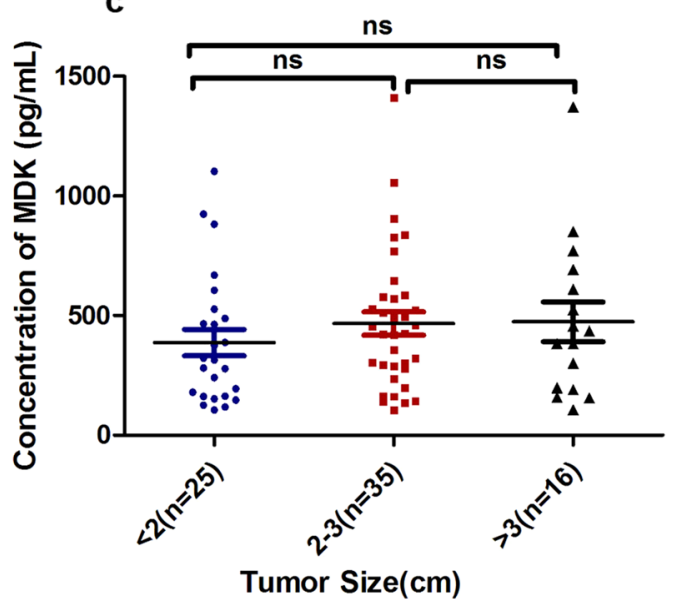

b

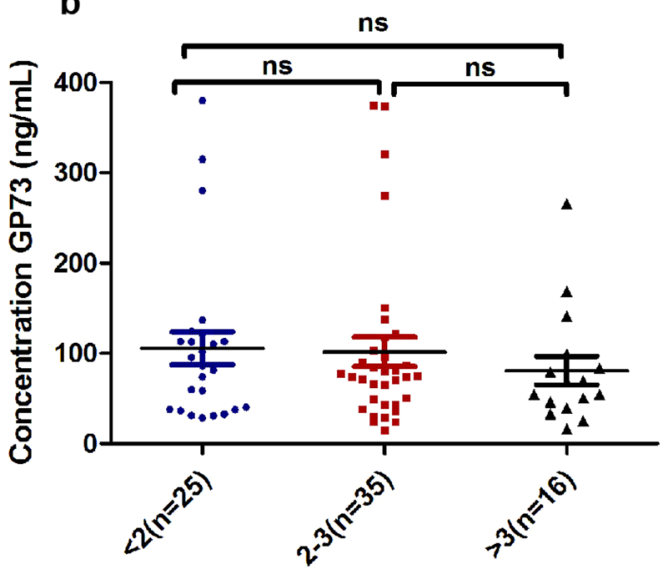

d

Tumor Size(cm)

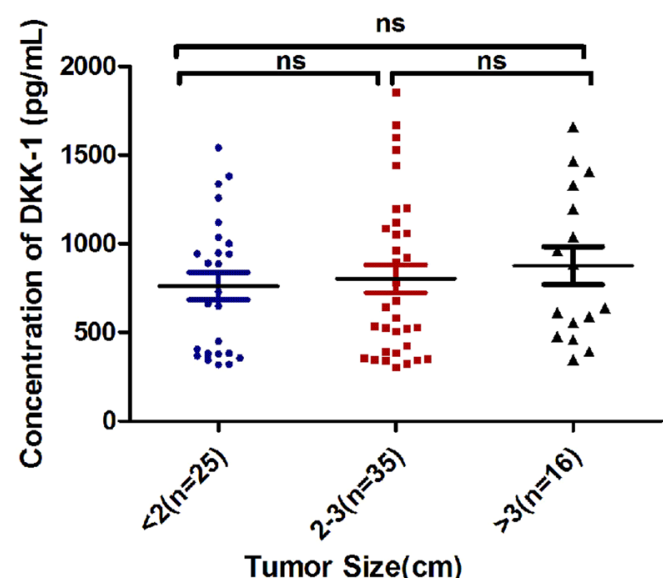

Figure 2. The correlation between serum levels and tumor size in HCC patients. 
a

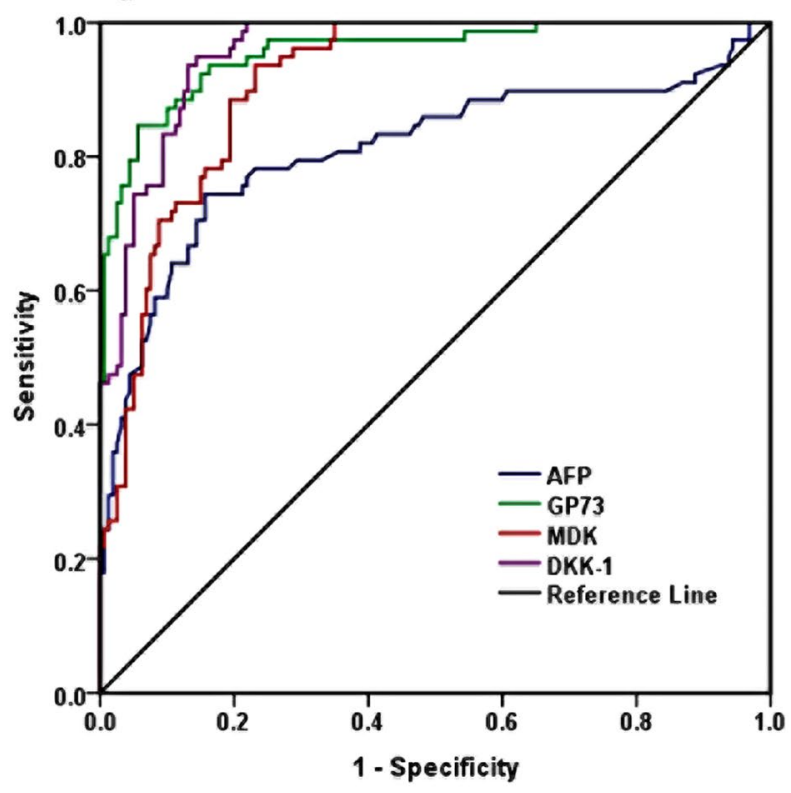

C

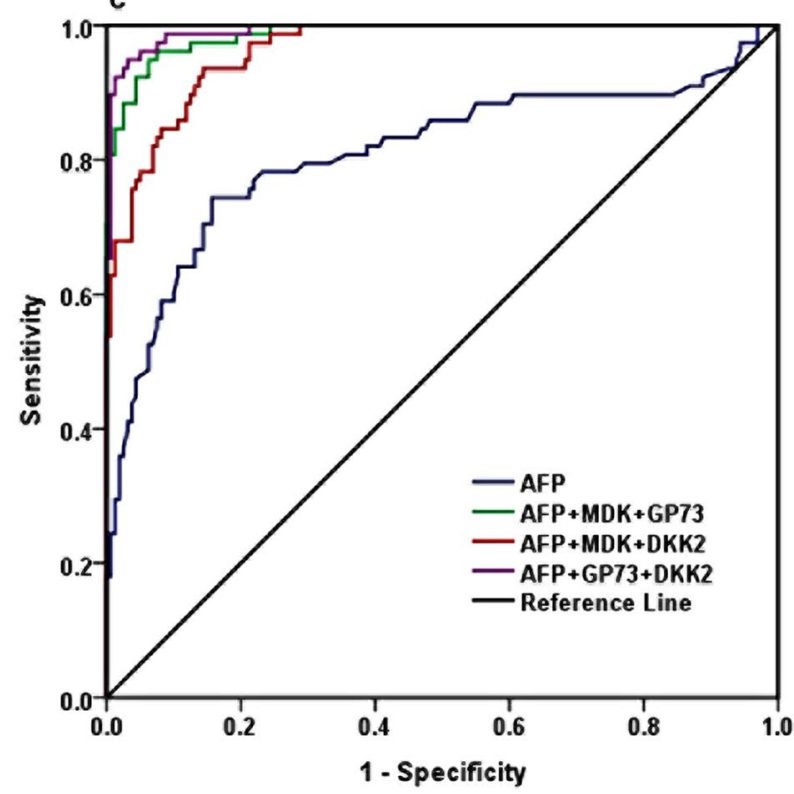

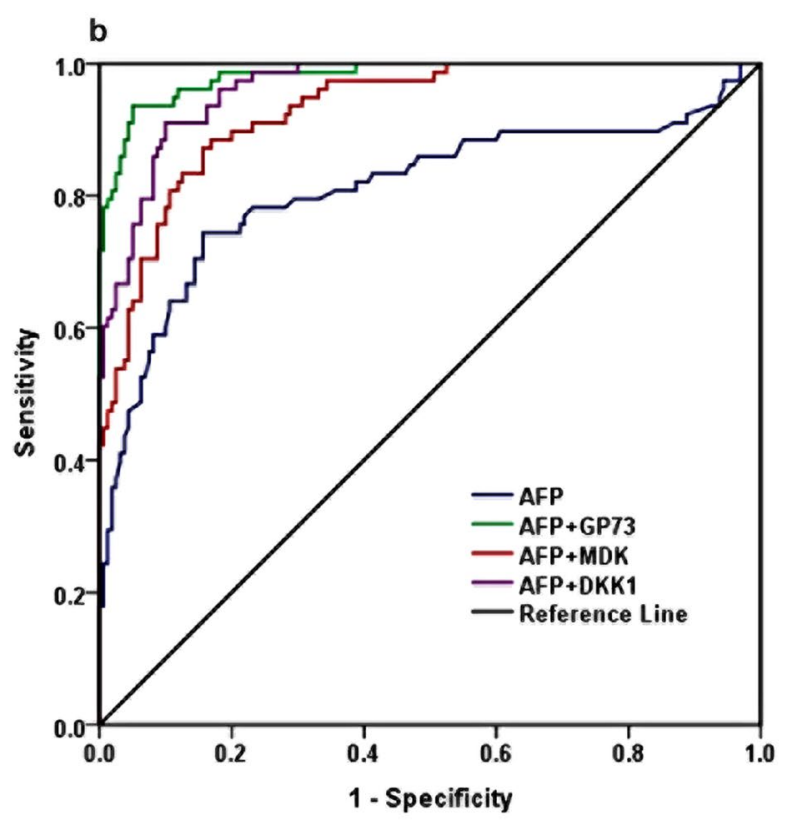

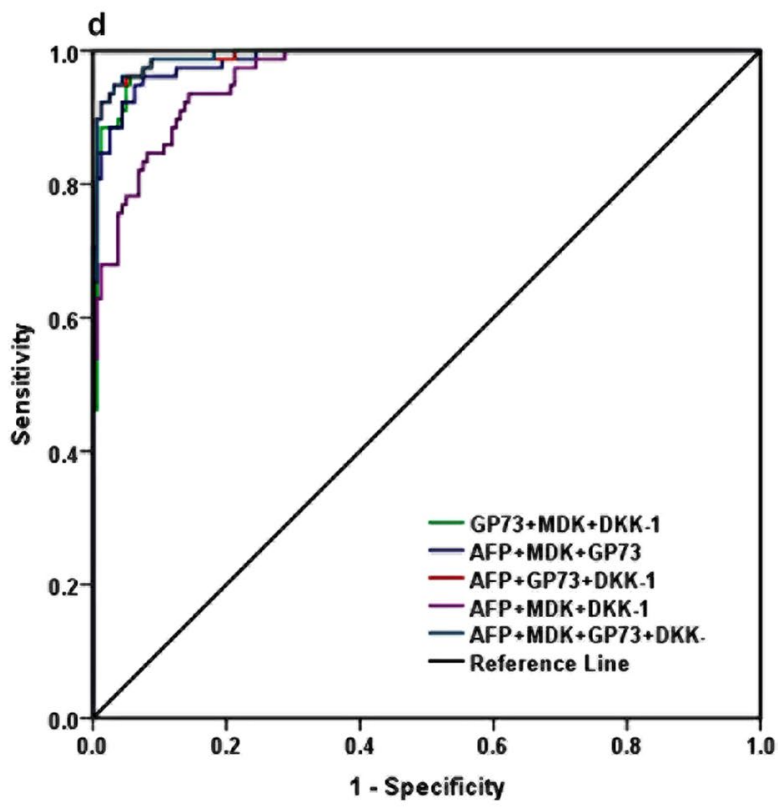

Figure 3. The optimal diagnostic cut off values of AFP, MDK, DKK-1, and GP73 were determined using ROC curve analysis.

DCP (des-gamma-carboxyprothrombin) which is also known as prothrombin induced by Vitamin K Absence II (PIVKA II). At the same time, there are several other new markers which are still under research including Dickkopf-1 (DKK1), Golgi Protein-73 (GP73), Glypican-3(GPC3), and different microRNAs (miRs) such as miR-29a, miR-29c, miR-133a, miR-143, miR-145, miR-192, and miR-505) ${ }^{7}$. The aim of the current study was to evaluate the possible role(s) of serum levels of GP73, MDK, DKK-1 proteins for early detection of HCC in chronic $\mathrm{HCV}$ infected patients, and to compare their diagnostic accuracy either with AFP or alone.

In the current study MDK levels were significantly higher in HCC group compared to the other studied groups. However there was no significant difference in the MDK protein expression level between the other two groups. This indicates that the diagnostic accuracy of MDK for HCC diagnosis is high and that it can also differentiate between HCC and non-HCC cases effectively irrespective of the presence of cirrhosis. This also shows that MDK may not be related to the inflammatory process and accordingly, it could be considered as an excellent, non- invasive diagnostic marker for HCC rather than the AFP. Our findings are in agreement with recently published reports from Europe and USA ${ }^{13,16,17}$. However, these data differs from other reports in literature which recommended the combination of MDK with AFP in HCC detection ${ }^{17}$. Our results also show that this combination of MDK with AFP enhances the sensitivity, specificity and accuracy, AUC and the 95\% CI. 


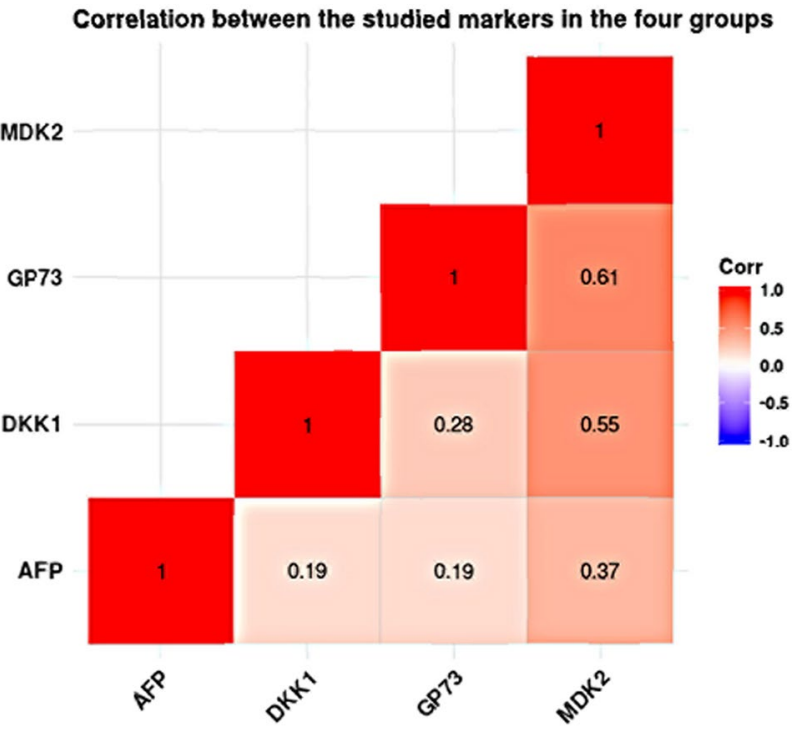

Figure 4. Correlation between the studies markers in the four groups.

\begin{tabular}{|c|c|c|c|c|c|c|c|c|c|}
\hline & $\begin{array}{l}\text { Sensitivity } \\
\text { (\%) }\end{array}$ & $\begin{array}{l}\text { Specificity } \\
(\%)\end{array}$ & \begin{tabular}{|l|} 
PPV \\
(\%)
\end{tabular} & \begin{tabular}{|l} 
NPV \\
$(\%)$
\end{tabular} & $\begin{array}{l}\text { Accuracy } \\
\text { (\%) }\end{array}$ & AUC & 95\%CI & $+\mathbf{L R}$ & $-\mathbf{L R}$ \\
\hline \multicolumn{10}{|l|}{ Single Marker } \\
\hline AFP & 74.4 & 84.4 & 69.9 & 87.1 & 81.1 & 0.81 & $0.74-0.88$ & 4.77 & 0.303 \\
\hline GP73 & 91 & 85 & 74.7 & 95 & 87 & 0.956 & $0.93-0.98$ & 6.10 & 0.110 \\
\hline MDK & 88.5 & 80.6 & 69 & 93.5 & 83.2 & 0.91 & $0.88-0.95$ & 4.60 & 0.14 \\
\hline DKK-1 & 93.6 & 86.9 & 77.7 & 96.5 & 89.08 & 0.956 & $0.93-0.98$ & 7.15 & 0.07 \\
\hline \multicolumn{10}{|l|}{ Double Markers } \\
\hline MDK + GP73 & 96.2 & 87.5 & 80.6 & 97.9 & 91.18 & 0.975 & $0.96-0.99$ & 7.69 & 0.04 \\
\hline MDK + DKK-1 & 91.0 & 85.0 & 79.3 & 94.0 & 88.7 & 0.956 & $0.93-0.98$ & 6.07 & 0.11 \\
\hline GP73 + DKK-1 & 97.4 & 93.1 & 87.36 & 98.7 & 94.5 & 0.987 & $0.98-0.99$ & 14.1 & 0.027 \\
\hline $\mathrm{AFP}+\mathrm{MDK}$ & 91.0 & 76.9 & 66.7 & 95.38 & 82.35 & 0.93 & $0.90-0.96$ & 3.94 & 0.12 \\
\hline AFP + DKK-1 & 91.0 & 90.0 & 82.56 & 95.39 & 90.76 & 0.963 & $0.94-0.98$ & 9.1 & 0.1 \\
\hline $\mathrm{AFP}+\mathrm{GP73}$ & 96.2 & 88.1 & 80.6 & 97.9 & 91.18 & 0.982 & $0.97-0.99$ & 8.08 & 0.043 \\
\hline \multicolumn{10}{|l|}{ Triple Markers } \\
\hline $\mathrm{AFP}+\mathrm{MDK}+\mathrm{GP73}$ & 96.2 & 92.5 & 86.2 & 98.01 & 93.7 & 0.987 & $0.98-1.0$ & 12.8 & 0.04 \\
\hline $\mathrm{AFP}+\mathrm{MDK}+\mathrm{DKK}-1$ & 93.6 & 85.6 & 76.04 & 96.48 & 88.2 & 0.964 & $0.94-0.98$ & 6.7 & 0.07 \\
\hline AFP + GP73 + DKK-1 & 98.7 & 91.2 & 86.5 & 99.3 & 94.5 & 0.99 & $0.98-1.0$ & 11.2 & 0.014 \\
\hline MDK + DKK-1 + GP73 & 98.7 & 91.2 & 86.5 & 91.2 & 94.5 & 0.99 & $0.98-1.0$ & 11.2 & 0.014 \\
\hline \multicolumn{10}{|l|}{ Quadruple Markers } \\
\hline $\mathrm{AFP}+\mathrm{GP73}+\mathrm{MDK}+\mathrm{DKK}-1$ & 98.7 & 91.2 & 84.6 & 99.3 & 93.7 & 0.99 & $0.99-1.0$ & 11.2 & 0.014 \\
\hline
\end{tabular}

Table 4. Diagnostic performance of AFP, MDK, DKK-1, and GP73 and their combinations for the diagnosis of HCC patients. PPV positive predictive value NPV negative predictive value AUC area under the curve LR likelihood ratio AFP: $\alpha$ fetoprotein, GP73: Golgi Protein 73, MDK: Midkine, DKK-1: Dickkopf-1 protein.

DKK-1 is another biomarker which was significantly higher in the HCC patients compared to other groups. However, there was no statistically significant difference in its levels across the non-HCC groups. This also confirms the important diagnostic role of DKK-1 in HCC patients and consequently it could be utilized in distinguishing HCC from other premalignant lesions as well as for different stages of chronic liver disease. This is in agreement with the study of Shen $e$ t al. ${ }^{18}$, and Geet $e t$ al. ${ }^{19}$, however they contrast with the previously reported data of Jang et al., 2016 who mentioned that AFP is still considered the most valuable and accurate marker for diagnosing HCC. In the contrary, our results contrast with those of Jang ES., $2011^{20}$ who showed that a combination of DKK-1 and AFP increases the sensitivity of DKK-1 but it reduces it's the specificity, accuracy, AUC, 95\% CI.

The GP73 is expressed in the biliary epithelial cells and hepatocytes and its increase is always related to the degree of liver injury. It has also been shown that, the amount of GP73 differs according to the degree of fibrosis or inflammation in the studied subjects ${ }^{21}$. We found that, serum GP73 was significantly elevated in HCC group compared to other groups though it was not significantly higher in the cirrhotic group compared to the non-cirrhotic group. This could be explained, at least partially by the fact that the amount of GP73 secreted by the 


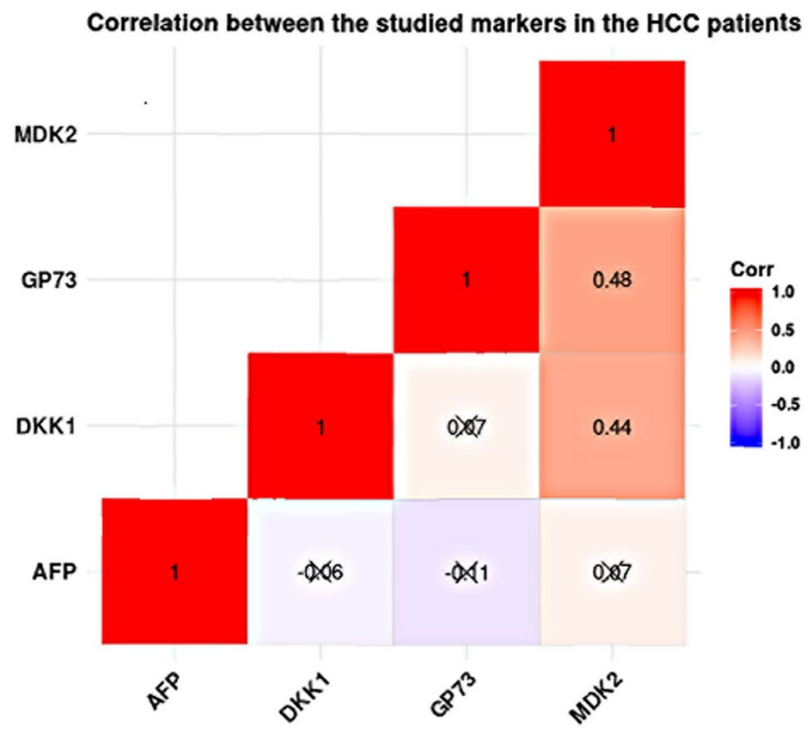

Figure 5. Correlation between the studies markers in the HCC patients.

\begin{tabular}{|c|c|c|c|}
\hline & MDK & DKK-1 & GP73 \\
\hline \multicolumn{4}{|c|}{ Overall population $(n=238)$} \\
\hline MDK & - & $\begin{array}{l}r=0.547 \\
P<0.001\end{array}$ & $\begin{array}{l}\mathrm{r}=0.612 \\
\mathrm{P}<0.001\end{array}$ \\
\hline DKK-1 & $\begin{array}{l}\mathrm{r}=0.547 \\
\mathrm{P}<0.001\end{array}$ & - & $\begin{array}{l}\mathrm{r}=0.282 \\
\mathrm{P}<0.001\end{array}$ \\
\hline GP73 & $\begin{array}{l}r=0.612 \\
P<0.001\end{array}$ & $\begin{array}{l}r=0.282 \\
P<0.001\end{array}$ & - \\
\hline \multicolumn{4}{|c|}{$\underline{\mathrm{HCC}}(\mathrm{n}=78)$} \\
\hline MDK & - & $\begin{array}{l}\mathrm{r}=0.441 \\
\mathrm{P}<0.001\end{array}$ & $\begin{array}{l}\mathrm{r}=0.482 \\
\mathrm{P}<0.001\end{array}$ \\
\hline DKK-1 & $\begin{array}{l}\mathrm{r}=0.441 \\
\mathrm{P}<0.001\end{array}$ & - & $\begin{array}{l}r=0.066 \\
P=0.565\end{array}$ \\
\hline GP73 & $\begin{array}{l}\mathrm{r}=0.482 \\
\mathrm{P}<0.001\end{array}$ & $\begin{array}{l}\mathrm{r}=0.066 \\
\mathrm{P}=0.565\end{array}$ & - \\
\hline
\end{tabular}

Table 5. Correlation analysis of studied markers in all studied groups and in HCC patients. $r=$ rho Pearson correlation coefficient, GP73: Golgi Protein 73, MDK: Midkine, DKK-1: Dickkopf-1 protein rho value range is $(0.2-0.5)$ between MDK, andGP73( $\mathrm{r}=0.612 ; \mathrm{p}<0.001 \&$ rho value $>0.5)$ (Supplement- 1$)$.

damaged hepatocytes and the activated stellate cells depend on the degree of cell injury and fibrosis. Thus, lower concentrations of GP73 in cirrhotic compared to the non-cirrhotic group might be related, at least partially, to the inability of the cirrhotic liver to produce proteins including the GP73 protein. This could support the important role of GP73 as a non-invasive serum biomarker for HCC diagnosis which high diagnostic accuracy. According to our data GP73showed better sensitivity and specificity for predicting HCC compared to AFP. Our data in this context support the previously published data of Mao Y. et al. ${ }^{22}$, Li B., et al..$^{23}$, and Chen, $2012^{18}$ who provided evidence that GP73 can act in combination with AFP in order to increase its specificity and sensitivity as well as its diagnostic accuracy. Taken together, this could highlight the benefit of using a combination of AFP and GP73 in order to improve the diagnostic accuracy compared to either of them alone. However, these findings contrasts with those of Özkan $\mathrm{H}$ et al. ${ }^{24}$, who found that GP73 has specificity and sensitivity lower than AFP as well as with Qiao Y. et al., 2014 and Liu T. ${ }^{25,26}$, who reported that GP73 is not a suitable marker for the detection of HCC because its elevation is not only related to HCC patients but also to cirrhotic patients.

According to our data a triple markers panel formed of GP73, DKK-1 and MDK have $100 \%$ sensitivity and $65.6 \%$ specificity, however the addition of AFP to this panel decreases the specificity to $54.4 \%$ with no added value to the sensitivity. The presence of Gp73 provides the best sensitivity and specificity in different double and/or triple combinations. We were not able to compare our markers' combinations with other studies since (1) we did not find previous studies in literature using the same markers panel used by our group and (2) there was no study in literature that compared between these three markers, and AFP, for the early detection of HCC (Supplement-3).

By comparing our four chosen markers (AFP, GP73,MDK and DKK-1), in terms of sensitivity, specificity, AUC, and accuracy, we found thatDKK-1 protein is the best single studied biomarker for detecting (diagnosing) HCC, which is in agreement with previous studies ${ }^{18-20}$. However, by making different markers' combinations (double, triple, and quadruple), we found that the sensitivity increases to approximately $100 \%$ with compromising 
the specificity which reaches approximately $50 \%$. Accordingly, we concluded that, the best double markers panel to be used includesGP73 and DKK-1 which provides $100 \%$ sensitivity, $74.4 \%$ specificity as well as AFP + GP73 which has $98.7 \%$ sensitivity and $76.2 \%$ specificity.

\section{Conclusion}

We conclude that serum levels of MDK, DKK-1, Gp73 were significantly higher in HCC patients compared to the non-HCC group. However, the best prediction value is provided by the combination of DKK-1 with Gp73as it showed high accuracy $(82.8 \%)$ and specificity $(74.4 \%)$. However, this preliminary study should be confirmed in a larger study including more patients from each studied group.

Received: 16 September 2019; Accepted: 2 March 2020;

Published online: 20 March 2020

\section{References}

1. Ferlay, J. et al. Cancer Incidence and Mortality Worldwide: IARC CancerBase No. 11. Lyon, France. GLOBOCAN v1.0. Int Agency Res Cancer. Available from: http://globocan.iarc.fr (2012).

2. El-Serag, H. B. Epidemiology of viral hepatitis and hepatocellular carcinoma. Gastroenterology 142, 1264-1273 (2012).

3. Colli, A. et al. Accuracy of Ultrasonography, Spiral CT, Magnetic Resonance, and Alpha-Fetoprotein in Diagnosing Hepatocellular Carcinoma: A Systematic Review: CME. Am. J. Gastroenterol. 101(3), 513-23 (2006).

4. Song, P., Tang, W. \& Kokudo, N. Serum biomarkers for early diagnosis of hepatocellular carcinoma. Transl. Gastrointest. Cancer 3(2), 103-5 (2014).

5. Li, D., Mallory, T. \& Satomura, S. AFP-L3: A new generation of tumor marker for hepatocellular carcinoma. Clin. Chim. Acta 313(1-2), 15-9 (2001).

6. Yao, M. et al. The Clinical Significance of GP73 in Immunologically Mediated Chronic Liver Diseases: Experimental Data and Literature Review. Clin. Rev. Allergy. (2018).

7. Qin, Q. F., Weng, J., Xu, G. X., Chen, C. M. \& Jia, C. K. Combination of serum tumor markers dickkopf-1, DCP and AFP for the diagnosis of primary hepatocellular carcinoma. Asian Pac. J. Trop. Med. 10(4), 409-13 (2017).

8. Hodeib, H., ELshora, O., Selim, A., Mohammed Sabry, N. \& Mohamed EL-ashry, H. Serum Midkine and Osteopontin Levels as Diagnostic Biomarkers of Hepatocellular Carcinoma. Electron. physician 9(1), 3492-8 (2017).

9. Maitra, A. \& Thuluvath, P. J. GP73 and liver disease: a (Golgi) complex enigma. Am. J. Gastroenterol. 99(6), 1096-8 (2004).

10. Liu, T. et al. Serum Golgi protein 73 is not a suitable diagnostic marker for hepatocellular carcinoma. Oncotarget 8(10), 16498-506 (2017).

11. Sato, N. et al. Wnt inhibitor Dickkopf-1 as a target for passive cancer immunotherapy. Cancer Res. 70(13), 5326-36 (2010).

12. Yan, Q. et al. Novel functional proteins interact with midkine in hepatic cancer cells. Hepatobiliary Pancreat. Dis. Int. 11(3), 272-7 (2012).

13. Vongsuvanh, R. et al. Midkine Increases Diagnostic Yield in AFP Negative and NASH-Related Hepatocellular Carcinoma. Avila MA, editor. PLoS One 11(5), e0155800 (2016).

14. Barreiros, A. P., Piscaglia, F. \& Dietrich, C. F. Contrast enhanced ultrasound for the diagnosis of hepatocellular carcinoma (HCC): Comments on AASLD guidelines. J. Hepatol. 57(4), 930-2 (2012).

15. Llovet, J. M. et al. Design and endpoints of clinical trials in hepatocellular carcinoma. J. Natl Cancer Inst. 100(10), 698-711 (2008).

16. Shaheen, K. Y. A., Abdel-Mageed, A. I., Safwat, E., Albreedy, A. M. The value of serum midkine level in diagnosis of hepatocellular carcinoma. Int. J. Hepatol. 1-5, (2015).

17. Zhu, W. W. et al. Evaluation of midkine as a diagnostic serum biomarker in hepatocellular carcinoma. Clin. Cancer Res. 19(14), 3944-54 (2013).

18. Shen, Q. et al. Serum DKK1 as a protein biomarker for the diagnosis of hepatocellular carcinoma: A large-scale, multicentre study. Lancet Oncol. 13(8), 817-26 (2012).

19. Ge, T. et al. Diagnostic values of alpha-fetoprotein, dickkopf-1, and osteopontin for hepatocellular carcinoma. Med. Oncol. 32 (3), 59 (2015).

20. Jang, E. S. et al. Diagnostic Performance of Alpha-Fetoprotein, Protein Induced by Vitamin K Absence, Osteopontin, Dickkopf-1 and Its Combinations for Hepatocellular Carcinoma. Plos One 11(3), e0151069 (2011)

21. Tian, L. et al. Serological AFP/Golgi protein 73 could be a new diagnostic parameter of hepatic diseases. Int. J. Cancer 129(8), 1923-31 (2011).

22. Mao, Y. et al. Golgi protein 73 (GOLPH2) is a valuable serum marker for hepatocellular carcinoma. Gut 59(12), 1687-93 (2010).

23. Li, B. et al. The Clinical Values of Serum Markers in the Early Prediction of Hepatocellular Carcinoma. Biomed. Res. Int. 5358615, (2017).

24. Özkan, H. et al. Diagnostic and Prognostic Validity of Golgi Protein 73 in Hepatocellular Carcinoma. Digestion 83(1-2), 83-8 (2011).

25. Qiao, Y. et al. Serum gp73 is also a biomarker for diagnosing cirrhosis in population with chronic HBV infection. Clin. Biochem. 47(16-17), 216-22 (2014).

26. Liu, T. et al. Serum Golgi protein 73 is not a suitable diagnostic marker for hepatocellular carcinoma. Oncotarget 8(10), 16498-506 (2017).

\section{Acknowledgements}

Margrit Saad for helping in drafting and arranging the manuscript. This work was supported by Science and Technology Development Fund \#5193 to ARNZ and Egypt National Cancer Institute.

\section{Author contributions}

A.Z. and M.K. designed the experiments, while R.T.Z., R.G. and A.A.B. performed all experiments. A.Z., E.S., R.H., M.M., O.A. and M.M.L. carried out interpretation of the results. A.Z. and M.K. conceived of the experiments. All authors participated in coordination and helped to draft the manuscript. All authors read and approved the final manuscript.

\section{Competing interests}

The authors declare no competing interests.

\section{Additional information}

Supplementary information is available for this paper at https://doi.org/10.1038/s41598-020-62051-6. 
Correspondence and requests for materials should be addressed to A.-R.N.

Reprints and permissions information is available at www.nature.com/reprints.

Publisher's note Springer Nature remains neutral with regard to jurisdictional claims in published maps and institutional affiliations.

(c) Open Access This article is licensed under a Creative Commons Attribution 4.0 International License, which permits use, sharing, adaptation, distribution and reproduction in any medium or format, as long as you give appropriate credit to the original author(s) and the source, provide a link to the Creative Commons license, and indicate if changes were made. The images or other third party material in this article are included in the article's Creative Commons license, unless indicated otherwise in a credit line to the material. If material is not included in the article's Creative Commons license and your intended use is not permitted by statutory regulation or exceeds the permitted use, you will need to obtain permission directly from the copyright holder. To view a copy of this license, visit http://creativecommons.org/licenses/by/4.0/.

(C) The Author(s) 2020 